\title{
Photophysical Properties of Self-Aggregated Porphyrin: Semiconductor Nanoassemblies
}

\author{
E. Zenkevich, ${ }^{1}$ T. Blaudeck, ${ }^{2}$ M. Abdel-Mottaleb, ${ }^{3}$ F. Cichos, ${ }^{2}$ A. Shulga, ${ }^{1}$ and C. von Borczyskowski ${ }^{3}$ \\ ${ }^{1}$ National Academy of Science, Frantsishak Skaryna Avenue, 220072 Minsk, Belarus \\ ${ }^{2}$ Photonics and Optical Materials Group, Center for Nanostructured Materials and Analytics (NanoMA), \\ Chemnitz University of Technology, 09107 Chemnitz, Germany \\ ${ }^{3}$ Optical Spectroscopy and Molecular Physics Group, Center for Nanostructured Materials and Analytics (NanoMA), \\ Chemnitz University of Technology, 09107 Chemnitz, Germany
}

Received 4 July 2005; Accepted 5 January 2006

Colloidal semiconductor nanocrystals from CdSe show photoluminescence quenching via titration with porphyrin derivatives. This quenching is an indication of the formation of nanoassemblies via surface attachment of pyridyl linker groups. As a consequence of the complex formation, dynamic and/or static interactions between QD and porphyrins are induced. Quenching efficiencies depend critically on sample stability, temperature, solvent, and electronic properties of the porphyrins. In order to optimize photoinduced dynamic processes these parameters have to be under control.

Copyright $\odot 2006$ E. Zenkevich et al. This is an open access article distributed under the Creative Commons Attribution License, which permits unrestricted use, distribution, and reproduction in any medium, provided the original work is properly cited.

\section{INTRODUCTION}

Narrowband fluorescence emission and colour tunability make semiconductor quantum dots (QDs) appropriate candidates for many future applications such as fluorescence labelling in biological relevant compounds [1]. Besides labelling experiments, photovoltaic applications are envisaged and those make use of nanostructured semiconductors coated with organic dye molecules [2]. Despite many reported experiments, little is known about the elementary interaction processes of QDs with their environment. For these nanoassemblies a microscopic description of the photophysical processes is also lacking. Combinations of singlemolecule/single-particle fluorescence microscopy with timeresolved and static optical spectroscopy allow for a novel detection scheme to unravel complex photoinduced dynamics both for isolated components of the envisaged nanoassemblies $[3,4]$ and the assemblies themselves. As an approach towards large-scale nanoassemblies we present a study on self-aggregation of organic molecules on QD surfaces and the respective photoinduced reaction dynamics. CdSe or $\mathrm{CdSe} / \mathrm{ZnS}$ core/shell QDs with diameters between 2 and $5 \mathrm{~nm}$ and a series of (pyridyl)-porphyrins were chosen as a model system, since pyridyl rings on the porphyrins allow complexation on to the QD surface. We could prove the complexation of the molecules to the surface by steadystate titration and time-resolved fluorescence measurements
$[5,6]$. Furthermore, decreased QD photoluminescence emission (PL) and enhanced porphyrin emission clearly indicate in several nanoassemblies an efficient fluorescence resonant energy transfer process (FRET). However, fluorescence quenching is also induced by processes other than FRET, which might be related to the inherent photoinduced blinking of single QDs, which we could for the first time describe systematically as being due to photoinduced self-trapping of charges in the dielectric medium of the environment of QDs [7].

To put these reported investigations of photodynamic processes of dye-semiconductor nanoassemblies on such a ground which is adequate for future applications, we investigated and present now a series of photophysical properties of nanoassemblies such as the influence of the chemical properties of various porphyrin systems, solvent polarity, sample preparation, long-time stability, and temperature.

\section{EXPERIMENTAL}

Trioctylphosphine oxide (TOPO)-capped highly monodisperse CdSe nanocrystals were prepared using a modification of the conventional organometallic synthesis [8]. In addition, surface passivation with an inorganic $\mathrm{ZnS}$ shell leads to an improvement of the room temperature quantum efficiency of the photoluminescence (PL) of CdSe up to 40-60\% [9]. Mainly one type of CdSe/ZnS nanocrystals was used in this 


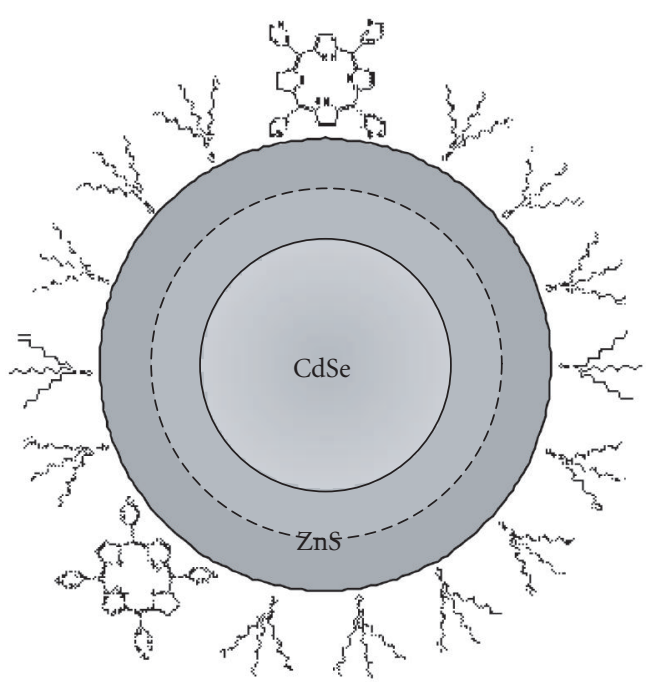

(a)

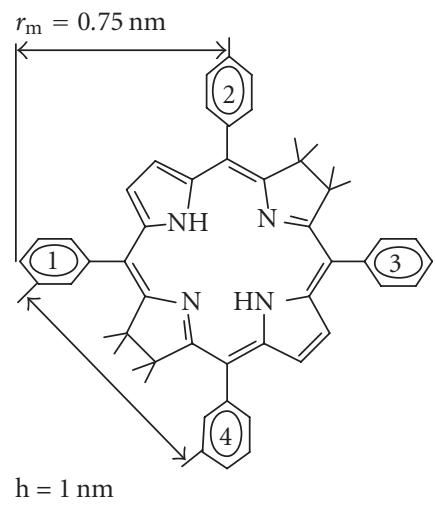

(b)

Scheme 1: Model of a YQD CdSe/ZnS nanoassembly with TOPO molecules and two porphyrins on the surface (a). Structure of tetrahydroporphyrin (THP) with a scheme for m-pyridyl substitution (b).

publication to form semiconductor nanoparticle-porphyrin composites in toluene and chloroform (Aldrich spectroscopic grade without further purification) at ambient temperature: "yellow" quantum dots (YQD) $(n=2 \mathrm{ZnS}$ monolayers) with the first excitonic maximum in absorption at $\lambda_{\max }=512 \mathrm{~nm}$. The CdSe diameter amounts to $d=2.5 \mathrm{~nm}$ for YQD. YQD has been provided by A. Rogach (LudwigMaximilians-University Munich, Germany) and D. Talapin (Hamburg Univeristy, Germany). Similar results have been obtained with QD provided by Evident Co., USA.

We have shown earlier [10] that selectively replacing phenyl rings in tetraphenyl porphyrins $\left(\mathrm{H}_{2} \mathrm{P}\right)$ with pyridyl rings opens the possibility of a controllable formation of multiporphyrin assemblies with $\mathrm{Zn}$-porphyrin dimers via a key-hole principle. In the present contributions experiments have been carried out using mesopyridyl-substituted porphyrins resulting in self-aggregated complexes with CdSe/ $\mathrm{ZnS}$. Scheme 1 shows as an example the structure and nomenclature of tetrahydro-porphyrin-free base (THP) with various numbers (from 1 to 4 ) of mesopyridyl rings differing in their relative positions with respect to the porphyrin macrocycle (opposite $(-)$ or adjacent $(\wedge)$ ) and with the position (ortho-, meta-, para-) of the nitrogen within the pyridyl ring. In addition, porphyrins with isopropylphenyl side chains were used to modify steric interactions with TOPO molecules as well as to improve $\mathrm{H}_{2} \mathrm{P}$ solubility. A schematic model for YQD and THP complexes based on our recent findings $[5,6]$ is also shown in Scheme 1 .

The experimental procedure for the formation of QD nanoassemblies has been reported recently [10]. The initial concentration of QD in toluene solutions (in a $10 \times 10 \mathrm{~mm}^{2}$ quartz cuvette) was in the range of $\mathrm{C}_{\mathrm{QD}} 0.5 \cdot 10^{-7} \mathrm{M}$. Porphyrins were added sequentially in steps of $10 \mu \mathrm{L}$ from a highly concentrated stock solution $\left(\mathrm{C}_{\text {por }}\left(\sim 8 \times 10^{-6} \mathrm{M}\right) /(4 \times\right.$ $\left.10^{-5} \mathrm{M}\right)$ ) up to the wanted molar ratios $x=\left[\mathrm{H}_{2} \mathrm{P}\right] /[\mathrm{QD}]$.

A spectrophotometer (Shimadzu UV-3101PC) was used for absorption measurements, whereas a Shimadzu RF5001PC spectrofluorimeter was used for fluorescence measurements. Temperatures have been varied in a temperaturecontrolled quartz cuvette.

\section{RESULTS AND DISCUSSION}

Because of the well-known phenomenon of quantum confinement of Wannier-Mott excitons in CdSe below its Bohr radius [11] $\left(r_{\mathrm{B}} \sim 50 \AA\right)$, colloidal semiconductor CdSe nanocrystals show size-dependent and thus tunable absorption and PL properties [12].

As has been shown recently [5] the photoluminescence of colloidal CdSe/Zn quantum dots in solution can be quenched by titration with organic dye molecules such as porphyrins. Similar effects have been observed also by other groups [13-15] and have been attributed to energy or charge transfer between QD and dye molecules. Since QDs are intrinsically subject to various effects which influence the quantum efficiency [7], quenching effects attributed to dye molecules have to be investigated with some precautions since these imposed effects will be in competition with other quenching processes which depend on QD synthesis, solvents, and capping ligands such as TOPO. Addition of dye molecules will therefore influence this delicate equilibrium. For this reason we investigated the influence of various parameters on the photoluminescence (PL) quenching efficiency and PL stability influenced by various porphyrin molecules. An example of absorption and PL spectra of YQD and $(\mathrm{m}-\mathrm{Pyr})_{4} \mathrm{THP}$ is shown in Figure 1, which clearly shows that there is a spectral overlap in the range of $525 \mathrm{~nm}$ which might give rise to FRET.

\subsection{Reproducibility}

Figure 2 shows the reproducibility of YQD quenching by $(\mathrm{m}-\mathrm{Pyr})_{4} \mathrm{H}_{2} \mathrm{P}$ for three different samples which have been prepared within about 1 year from the same stock solution of YQD in toluene. In a recent publication [5] we have extensively shown that the quenching of PL is accompanied by a concentration-dependent increase of the $\mathrm{H}_{2} \mathrm{P}$ fluorescence. However, a quantitative comparison of quenching and FRET 


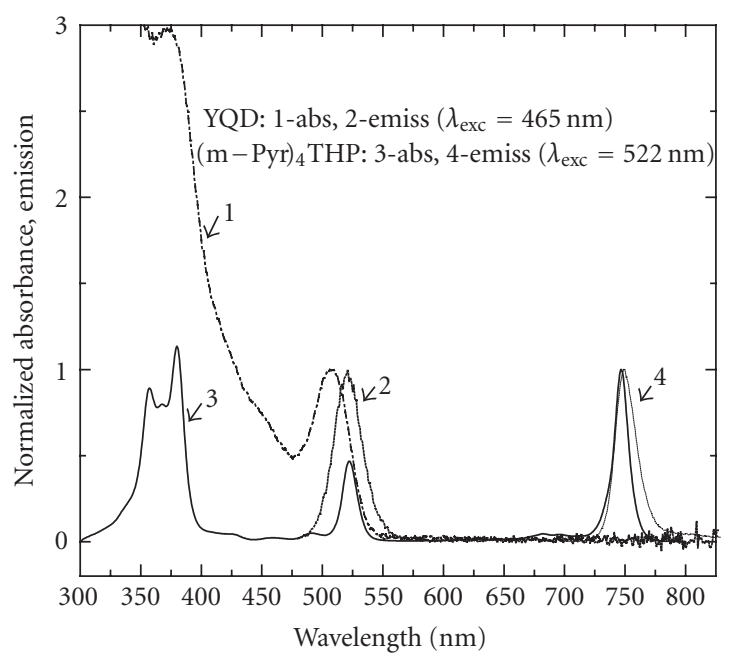

FIgUre 1: Absorption (1) and PL (2) spectrum of YQD in toluene. Absorption (3) and fluorescence (4) spectrum of THP in toluene.

has shown [5], that at least in the core/shell QDs FRET is considerably smaller than expected from PL quenching, which demands for the presence of additional yet unidentified quenching processes. This seems to be different as compared to other recently reported systems which reveal only FRET [9].

The comparison of the three different samples in Figure 2 shows that basically similar quenching efficiencies can be observed but that especially at molar ratios above $x \approx 2$ noticeable deviations occur. It has to be mentioned that curve ( $\boldsymbol{\Lambda})$ which shows the most effective quenching has been investigated on a more extended time scale than the other two. As will be discussed, the reproducibility will strongly depend on the time scale on which experiments are performed.

\subsection{Stability}

In order to investigate the influence of the experimental time scale we have (as shown in Figure 3) monitored the quenching efficiency as a function of waiting time at various molar ratios. The quenching efficiency has been monitored for a fixed initial value of $(x)$ while waiting after the initial titration step. During that corresponding waiting time, the sample has been kept in the dark. It is evident from Figure 3 that the quenching efficiency increases in the course of time. After an initial quenching which is present immediately after the initial titration step on a time scale shorter than 2 minutes, an increase in quenching depending on $(x)$ is observed on time scales of 15 to 30 minutes while becoming shorter at higher $x$ values (Figure 3(a)). However, at times as long as up to 100 hours quenching efficiency is still increasing as can be seen from Figure 3(b). These measurements show that a delicate equilibrium among known and unknown quenching mechanisms exists which makes it difficult to define a quenching efficiency accurately. This equilibrium is probably due to competition of intrinsic and extrinsic quenching

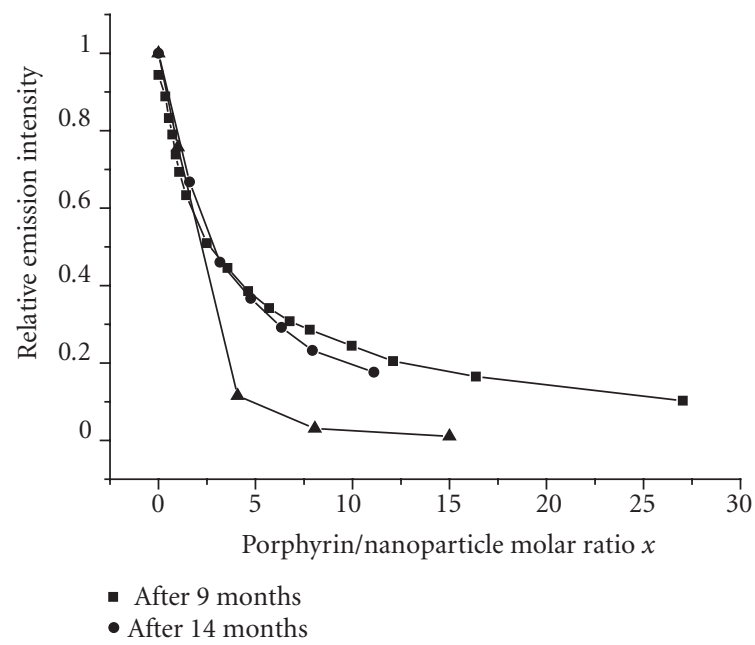

Figure 2: PL quenching of YQD by $(\mathrm{m}-\mathrm{Pyr})_{4} \mathrm{H}_{2} \mathrm{P}$ in toluene. Data are obtained for 3 different samples from the same YQD stock solution after 9 months $(\boldsymbol{\square})$ and after 14 months $(\bullet)$.

centers on the surface of a QD, the equilibrium between the ligands (TOPO) on the surface or in the solution, and the titrated dye molecules. Probably the initial fast step is the occupation of free coordination sites on the QD surface while the long-time behaviour is due to the establishment of an equilibrium between dyes and TOPO and/or QD surface reorganisation.

\subsection{Solvent dependence}

Consequently such an equilibrium will depend on the solvent as is shown in Figure 4 for the two solvents toluene and chloroform. The influence of solvent composition has also been demonstrated recently [5]. Since toluene is less polar than chloroform, obviously a better solvation hinders the formation of nanoassemblies as seen by a reduced PL quenching.

\subsection{Temperature dependence}

Also temperature has an impact on the quenching efficiency as is shown in Figure 5. For a given QD or QD-dye nanoassembly, the QD PL intensity has a maximum near $T=273 \mathrm{~K}$. The intensity is decreasing both towards higher and lower temperatures. When proceeding to higher temperatures, only part of the intensity decrease is reversible upon lowering temperature again. We could not detect any noticeable difference between QD or QD-dye nanoassemblies.

A clear understanding of the temperature dependence will need more elaborate experiments. However, a few conclusions can already be drawn from the present results. With respect to possible processes responsible for the PL quenching, one can safely assume that FRET will not depend drastically on temperature since spectral overlaps did not change noticeable in that temperature range. Also a drastic change of structure is not to be expected [5]. Moreover, the temperature dependence of PL is almost not influenced by the 


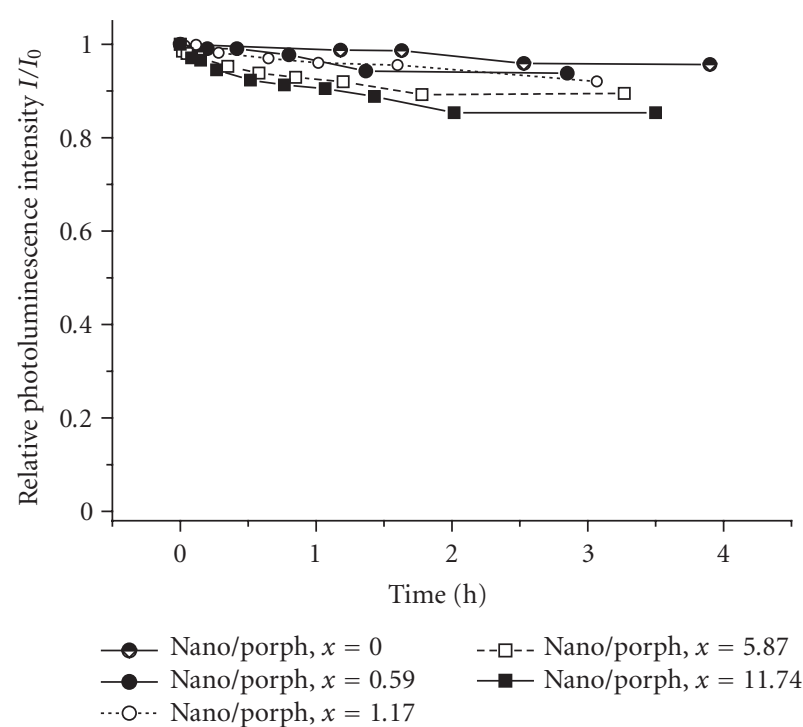

(a)

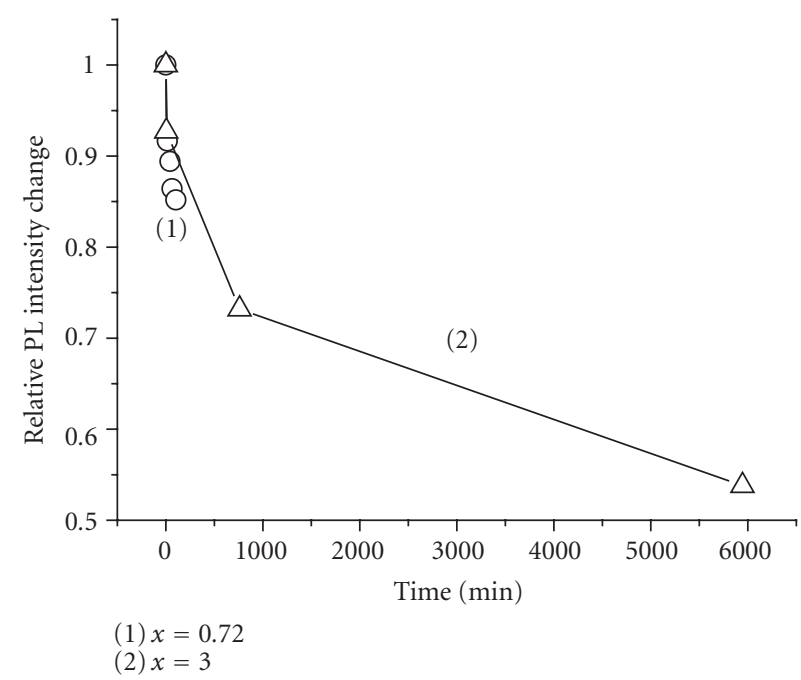

(b)

Figure 3: (a) PL quenching of Hops Yellow (CdSe/ZnS QD, Evident) in toluene as a function of waiting time after one titration step by $(\mathrm{m}-\mathrm{Pyr})_{4} \mathrm{H}_{2} \mathrm{P}$ at various molar ratios $(x)$. All data have been normalized to 1 at $t=0$. (b) PL quenching of Lace Placid Blue (CdSe QD, Evident) in toluene as function of waiting time after one titration step by $(\mathrm{m}-\mathrm{Pyr})_{4} \mathrm{H}_{2} \mathrm{P}$ at $x=0.72(1)$ and $x=3(2)$. Data have been normalized to 1 at $t=0$.

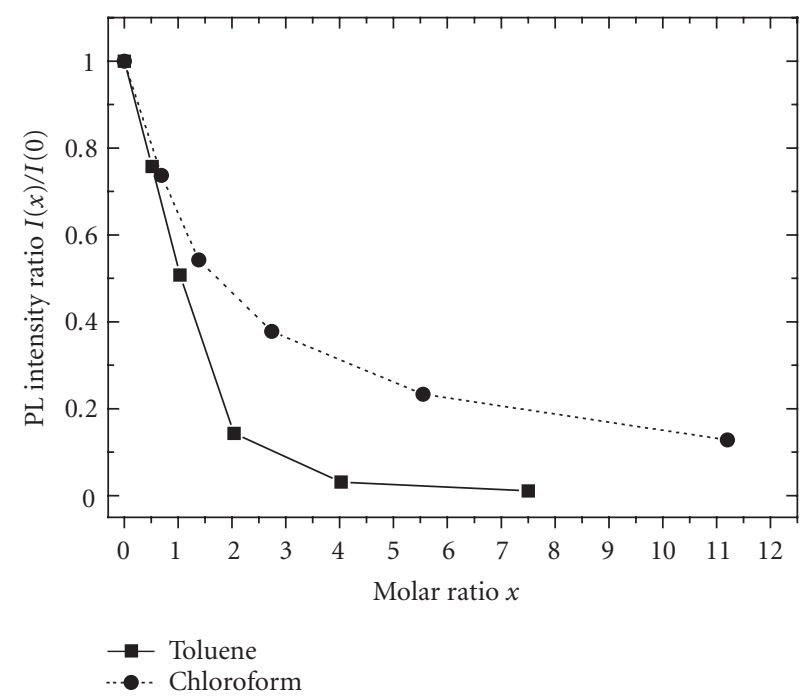

FIGURE 4: PL quenching of YQD by $(\mathrm{m}-\mathrm{Pyr})_{4} \mathrm{H}_{2} \mathrm{P}$ in toluene and chloroform.

formation of nanoassemblies. This implies that the temperature dependence is obviously a property of the QDs itself. Recently Meijerink et al. [16] published temperaturedependent data on related QDs and explained them in terms of a temperature-dependent interrelationship of QD core and surface states. Since surface states might be related to charged states (vide infra), this interrelationship will depend on solvent polarity and viscosity [17], which are both strongly temperature dependent. We have independently shown [18] that QD PL intensity and stability depend on the water content of the solvent. This might explain the PL maximum at about $273 \mathrm{~K}$, at which water might freeze out on the QD surface. A clear understanding of the temperature dependence demands for an extensive study of all these delicate interrelationships which is far beyond the scope of the present publication.

\subsection{Electronic and geometric properties}

In case that photoinduced fluorescence resonant energy transfer (FRET) or charge transfer are responsible for the PL quenching via porphyrin attachment, the quenching efficiency should depend drastically on (i) variations in geometry via space filling substituents, (ii) redox potentials of charge donors and acceptors, and (iii) spectral overlap of PL donor emission and acceptor adsorption. To investigate these aspects we have prepared nanoassemblies (isoPrPh $)_{2}$ $(\mathrm{m} \wedge \mathrm{Pyr})_{2} \mathrm{H}_{2} \mathrm{P}$ with spacefilling isopropyl side groups (case (i) ) and fluorinated phenyl groups in form of $(5 \mathrm{~F}-\mathrm{Ph})_{2}$ $(\mathrm{m} \wedge \mathrm{Pyr})_{2} \mathrm{H}_{2} \mathrm{P}$. Fluorination results in a drastically changed redox potential [19] (case (ii)). A comparison of PL quenching of fluorinated and isopropyl-substituted $(\mathrm{m} \wedge \mathrm{Pyr})_{2} \mathrm{H}_{2} \mathrm{P}$ with $(\mathrm{p} \wedge \mathrm{Pyr})_{2} \mathrm{H}_{2} \mathrm{P}$ is shown in Figure 6 . With respect to absorption and fluorescence spectra, all investigated $(\mathrm{Pyr})_{2} \mathrm{H}_{2} \mathrm{P}$ compounds are nearly indistinguishable.

While the quenching efficiency is nearly the same for (isoPrPh $)_{2} \mathrm{H}_{2} \mathrm{P}$ and $(\mathrm{p} \wedge \mathrm{Pyr})_{2} \mathrm{H}_{2} \mathrm{P}$, it is less effective for $(5 \mathrm{~F}-\mathrm{Ph})_{2}(\mathrm{~m} \wedge \mathrm{Pyr})_{2} \mathrm{H}_{2} \mathrm{P}$. (Para- and meta $\mathrm{H}_{2} \mathrm{P}$ compounds show nearly the same quenching efficiencies [5].) Thus case (i) confirms our earlier findings [5] that $(\mathrm{m} \wedge \mathrm{Pyr}) \mathrm{H}_{2} \mathrm{P}$ anchors with two pyridyl groups nearly perpendicular on the QD surface. Since $\mathrm{H}_{2} \mathrm{P}$ is slightly larger than the TOPO 


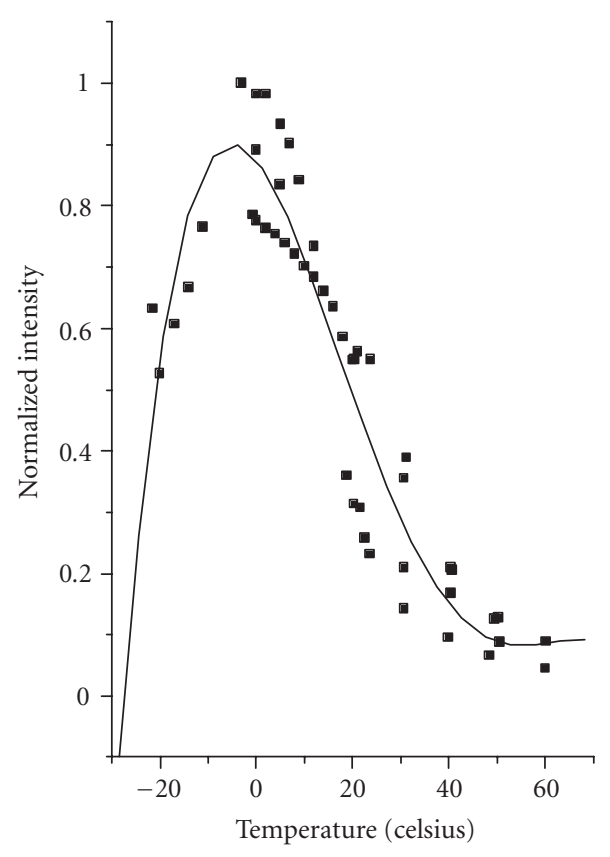

FIGURE 5: Temperature dependence of normalized YQD PL in toluene as a function of temperature. Data have been collected from several measurements on samples with and without titration with $(\mathrm{m}-\mathrm{Pyr})_{4} \mathrm{H}_{2} \mathrm{P}$. Temperature cycles are subject to irreversible PL losses. The line is merely a guideline for the eye.

ligands (see Scheme 1), the isopropyl-substituted phenyl rings are outside the effective TOPO shell which results in negligible spatial hindrance due to these isopropyl groups.

In case of supramolecular porphyrin assemblies, it has been shown [19] that fluorinated $\mathrm{H}_{2} \mathrm{P}$ compounds withdraw strongly electrons from a suitable molecular counterpart even at very low temperatures. Considering our model for the structure of the nanoassembly [5], the fluorinated phenyl groups are not in direct contact with the QD surface which is somewhat different from the recently reported supramolecular assemblies $[10,19]$. In case that electron transfer from QD to fluorinated $\mathrm{H}_{2} \mathrm{P}$ would be effective, we would expect an even stronger PL quenching as compared to $(\mathrm{m} \wedge \mathrm{Pyr})_{2} \mathrm{H}_{2} \mathrm{P}$, which is while inspecting Figure 6 not the case, where the corresponding quenching is even less. By this observation, a photoinduced electron transfer from QD to $\mathrm{H}_{2} \mathrm{P}$ is ruled out.

Two reasons might account for the reduced quenching efficiency, that is firstly, a reduced complexation due to the more spacious fluor atoms which is with respect to the findings for the isopropyl-substituted $\mathrm{H}_{2} \mathrm{P}$ very unlikely. Secondly, since we have found photoinduced energy transfer from YQD to $(\mathrm{m} \wedge \mathrm{Pyr})_{2} \mathrm{H}_{2} \mathrm{P}[5]$, we can not neglect the presence of energy transfer for the fluorinated compound too. However, also in our recent findings [5], it became evident that energy transfer might be too small (in several cases) to explain the effective quenching. Therefore, we hypothetically assume that hole (instead of electron) and energy transfers might be competing processes which both would result in QD PL quenching. On the $\mathrm{H}_{2} \mathrm{P}$ acceptor side energy trans-

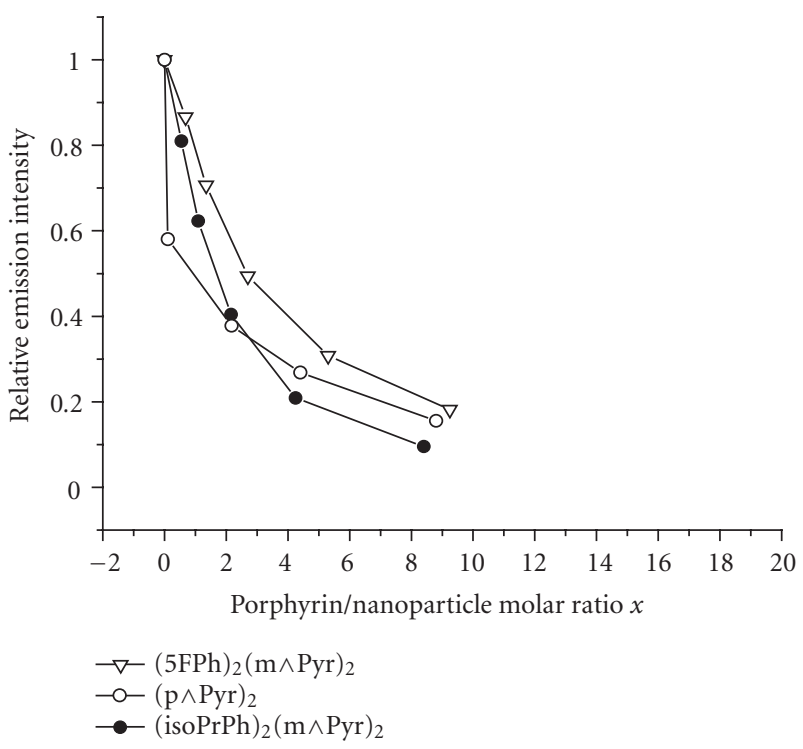

FIGURE 6: PL quenching of YQD in toluene as a function of $(x)$ for 3 different porphyrins.

fer will result in a fluorescence increase while hole (or electron) transfer will result in a diminished $\mathrm{H}_{2} \mathrm{P}$ fluorescence. Depending on the relative ratios of these two effects, $\mathrm{H}_{2} \mathrm{P}$ fluorescence and PL quenching will depend on the redox potentials of $\mathrm{H}_{2} \mathrm{P}$. Assuming that $(5 \mathrm{~F}-\mathrm{Ph})_{2}(\mathrm{~m} \wedge \mathrm{Pyr})_{2} \mathrm{H}_{2} \mathrm{P}$ is in principal suited to accept an electron from the conduction band of CdSe, this would result in an increase of QD $\mathrm{PL}$ quenching for $(5 \mathrm{~F}-\mathrm{Ph})_{2}(\mathrm{~m} \wedge \mathrm{Pyr})_{2} \mathrm{H}_{2} \mathrm{P}$ as compared to $(\mathrm{m} \wedge \mathrm{Pyr})_{2} \mathrm{H}_{2} \mathrm{P}$, which is, however, not observed. Hole transfer from $\mathrm{QD}$ to $\mathrm{H}_{2} \mathrm{P}$, on the other hand, might be an unidentified additional quenching process. With this assumption of hole transfer, it could be explained why the quenching efficiency is less than $(5 \mathrm{~F}-\mathrm{Ph})_{2}$, because $(\mathrm{m} \wedge \mathrm{Pyr})_{2} \mathrm{H}_{2} \mathrm{P}$ is an effective electron acceptor but not an effective electron donor (hole acceptor). Hole transfer has been observed for $n$-butylamines assembled on uncapped CdSe QD [15].

However, whether such processes also apply to CdSe/ZnS remains an open question since both electron and hole transfers should, due to the $\mathrm{ZnS}$ shell barrier, have to occur via tunneling over a relatively long distance (larger than $1 \mathrm{~nm}$ ). The same would apply to Dexter-type energy transfer since both types of interactions rely on a direct overlap of electronic wave functions. Since this overlap decays exponentially with distance, they are processes probably to be ruled out. Therefore this still open question of additional quenching processes has to be investigated in the future considering the impact of surface states.

Also from recent results, it is plausible [5] that chargedependent quenching processes might be involved since the quenching efficiency is considerably increased upon increasing the polarity of the solvent by adding acetone. Complexation of pyridine itself has, at comparable molar ratios, an almost negligible influence on the PL quenching [5]. This polarity dependence points towards charged surface states as has also been pointed out recently [17]. 


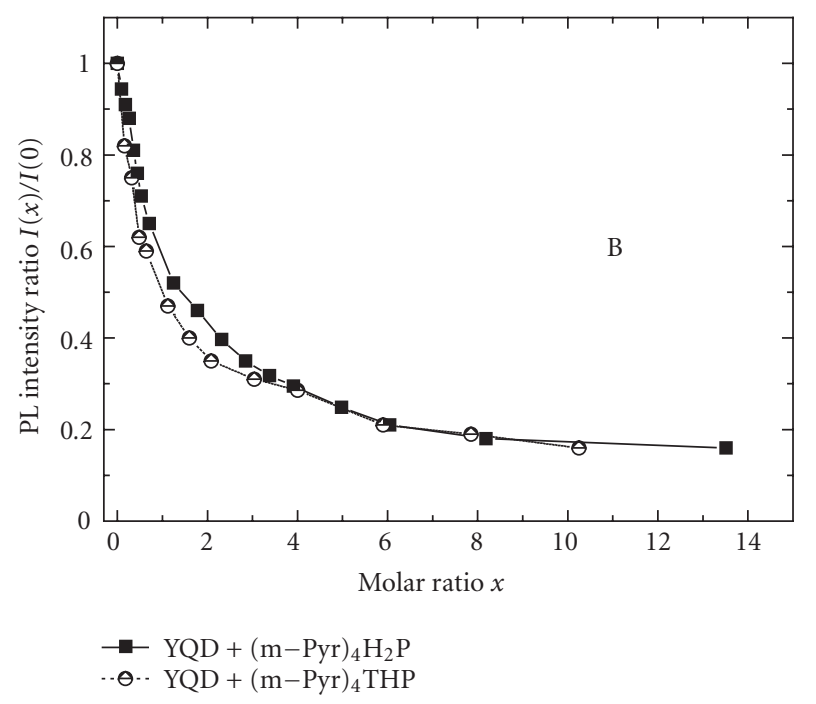

Figure 7: Comparison of PL quenching of YQD for $(\mathrm{m}-\mathrm{Pyr})_{4} \mathrm{H}_{2} \mathrm{P}$ and $(\mathrm{m}-\mathrm{Pyr})_{4}$ THP as a function of the molar ratio $(x)$.

Replacing $(\mathrm{m} \wedge \mathrm{Pyr})_{4} \mathrm{H}_{2} \mathrm{P}$ by $(\mathrm{m} \wedge \mathrm{Pyr})_{4} \mathrm{THP}$, where tetrahydro-porphyrin (THP) replaces $\mathrm{H}_{2} \mathrm{P}$, the conjugation of the pyrrole ring is changed which results in a considerable red shift of the corresponding fluorescence emission from about $650 \mathrm{~nm}$ to about $750 \mathrm{~nm}$. This results in a change of the spectral overlap (case (iii)), as can be seen from Figure 1, where the Q-Band absorption at $522 \mathrm{~nm}$ exhibits, as compared to $\mathrm{H}_{2} \mathrm{P}$, a changed spectral overlap with the YQD PL. This should change FRET processes considerably. However, as can be seen from Figure 7, the quenching efficiency remains nearly the same. We take this as a further indication that at least one additional quenching mechanism besides FRET has to be considered in order to describe the PL quenching efficiency properly.

\section{CONCLUSIONS}

We could show that various external parameters can control the photoluminescence efficiency (and PL quenching) of CdSe quantum dots. The data show that PL quenching is reproducible but depends on reorganization processes of the ligand shell. The final equilibrium depends on solvent composition and temperature. Also intrinsic properties of the porphyrins such as geometry and electronic structure have an impact on PL quenching. Whereas geometric effects are of minor importance, the electronic properties have noticeable impact on the QD PL. This indicates that-besides FRET-additional not yet identified mechanisms are competitive quenching routes. Charged surface states controlled by the molecular assembly process have to be taken into account as one major reason for non-FRET processes.

\section{ACKNOWLEDGMENTS}

YQD was given by Dr. A. Rogach (Ludwig-MaximiliansUniversity Munich, Germany) and Dr. D. Talapin (Hamburg
University, Germany), which is gratefully acknowledged. Financial support has been provided by the graduate college Accumulation of Single Molecules to Nanostructures of the German Science Foundation (T. Blaudeck), the VWfoundation within the research program "Physics, Chemistry and Biology with Single Molecules" (M. Abdel-Mottaleb), the Belarus National Program of Basic and Oriented Research (Nanotech. 5.17), and INTAS Grant no. 03-50-4540. E. Zenkevich is also thankful to the German Academic Research Service for a DAAD Research Grant (2003).

\section{REFERENCES}

[1] A. Sukhanova, J. Devy, L. Venteo, et al., "Biocompatible fluorescent nanocrystals for immunolabeling of membrane proteins and cells," Analytical Biochemistry, vol. 324, no. 1, pp. 6067, 2004.

[2] S. Pelet, J.-E. Moser, and M. J. Grätzel, "Cooperative effect of adsorbed cations and iodide on the interception of back electron transfer in the dye sensitization of nanocrystalline $\mathrm{TiO}_{2}$," Journal of Physical Chemistry B, vol. 104, no. 8, pp. 1791-1795, 2000.

[3] Ph. Tamarat, A. Maali, B. Lounis, and M. Orrit, "Ten years of single-molecule spectroscopy," Journal of Physical Chemistry A, vol. 104, no. 1, pp. 1-16, 2000.

[4] M. Nirmal, B. O. Dabbousi, M. G. Bawendi, et al., "Fluorescence intermittency in single cadmium selenide nanocrystals," Nature, vol. 383, pp. 802-804, 1996.

[5] E. Zenkevich, F. Cichos, A. Shulga, E. Petrov, T. Blaudeck, and C. von Borczyskowski, "Nanoassemblies designed from semiconductor quantum dots and molecular arrays," Journal of Physical Chemistry B, vol. 109, no. 18, pp. 8679-8692, 2005.

[6] E. Zenkevich, A. Shulga, T. Blaudeck, F. Cichos, and C. von Borczyskowski, Physics, Chemistry and Applications of Nanostructures: Reviews and Short Notes to Nanomeeting-2005, edited by V. I. Borisenko, S. V. Gaponenko, and V. S. Gurin, World Scientific, Hackensack, NJ, USA, 2005.

[7] A. Issac, C. von Borczyskowski, and F. Cichos, "Correlation between photoluminescence intermittency of CdSe quantum dots and self-trapped states in dielectric media," Physical Review B, vol. 71, p. 16130(R), 2005.

[8] A. M. Kapitonov, A. P. Stupak, S. V. Gaponenko, E. P. Petrov, A. L. Rogach, and A. Eychmüller, "Luminescence properties of thiol-stabilized CdTe nanocrystals," Journal of Physical Chemistry B, vol. 103, no. 46, pp. 10109-10113, 1999.

[9] B. R. Fischer, H.-J. Eisher, N. E. Stott, and M. G. Bawendi, "Emission intensity dependence and single-exponential behavior in single colloidal quantum dot fluorescence lifetimes," Journal of Physical Chemistry B, vol. 108, no. 1, pp. 143-148, 2004.

[10] E. Zenkevich and C. von Borczyskowski, Handbook of Polyelectrolytes and Their Applications, vol. 2, chapter 11, American Scientific, Stevenson Ranch, Calif, USA, 2002.

[11] G. Schmid, Ed., Nanoparticles: From Theory to Applications, Wiley-VCH, Weinheim, Germany, 2004.

[12] L. E. Brus, "Quantum crystallites and nonlinear optics," Applied Physics A: Materials Science \& Processing, vol. 53, no. 6, pp. 465-474, 1991.

[13] A. R. Clapp, I. L. Medintz, J. M. Mauro, B. R. Fisher, M. G. Bawendi, and H. Mattoussi, "Fluorescence resonance energy transfer between quantum dot donors and dye-labeled protein acceptors," Journal of the American Chemical Society, vol. 126, no. 1, pp. 301-310, 2004. 
[14] C. Burda, T. C. Green, S. Link, and M. A. El-Sayed, "Electron shuttling across the interface of CdSe nanoparticles monitored by femtosecond laser spectroscopy," Journal of Physical Chemistry B, vol. 103, no. 11, pp. 1783-1788, 1999.

[15] C. Landes, C. Burda, M. Braun, and M. A. El-Sayed, "Photoluminescence of CdSe nanoparticles in the presence of a hole acceptor: $n$-butylamine," Journal of Physical Chemistry B, vol. 105, no. 15, pp. 2981-2986, 2001.

[16] S. F. Wuister, A. van Houselt, C. de Mello Donegá, D. Vanmaekelbergh, and A. Meijerink, "Temperature antiquenching of the luminescence from capped CdSe quantum dots," Angewandte Chemie International Edition, vol. 43, no. 23, pp. 30293033, 2004.

[17] J. Tang and R. A. Marcus, "Mechanisms of fluorescence blinking in semiconductor nanocrystal quantum dots," The Journal of Chemical Physics, vol. 123, p. 54704, 2005.

[18] T. Blaudeck, thesis, Tu Chemnitz, Chemnitz, Germany, in preparation.

[19] E. Zenkevich, C. von Borczyskowski, and A. Shulga, "Structure and excited state properties of multiporphyrin arrays formed by supramolecular design," Journal of Porphyrins \& Phthalocyanines, vol. 7, no. 11-12, pp. 731-754, 2003. 


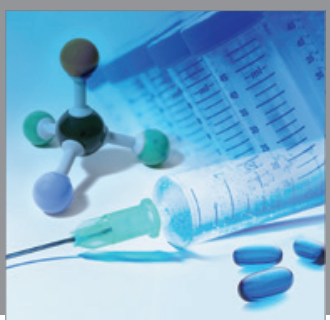

International Journal of

Medicinal Chemistry

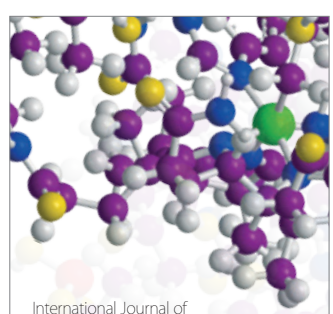

Carbohydrate Chemistry

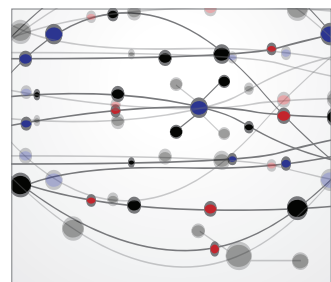

The Scientific World Journal
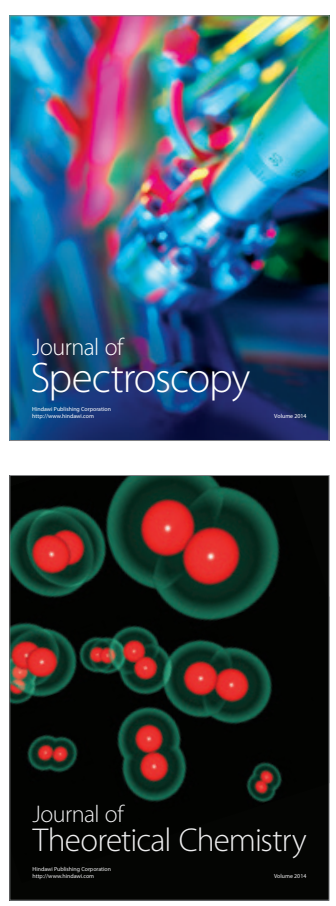
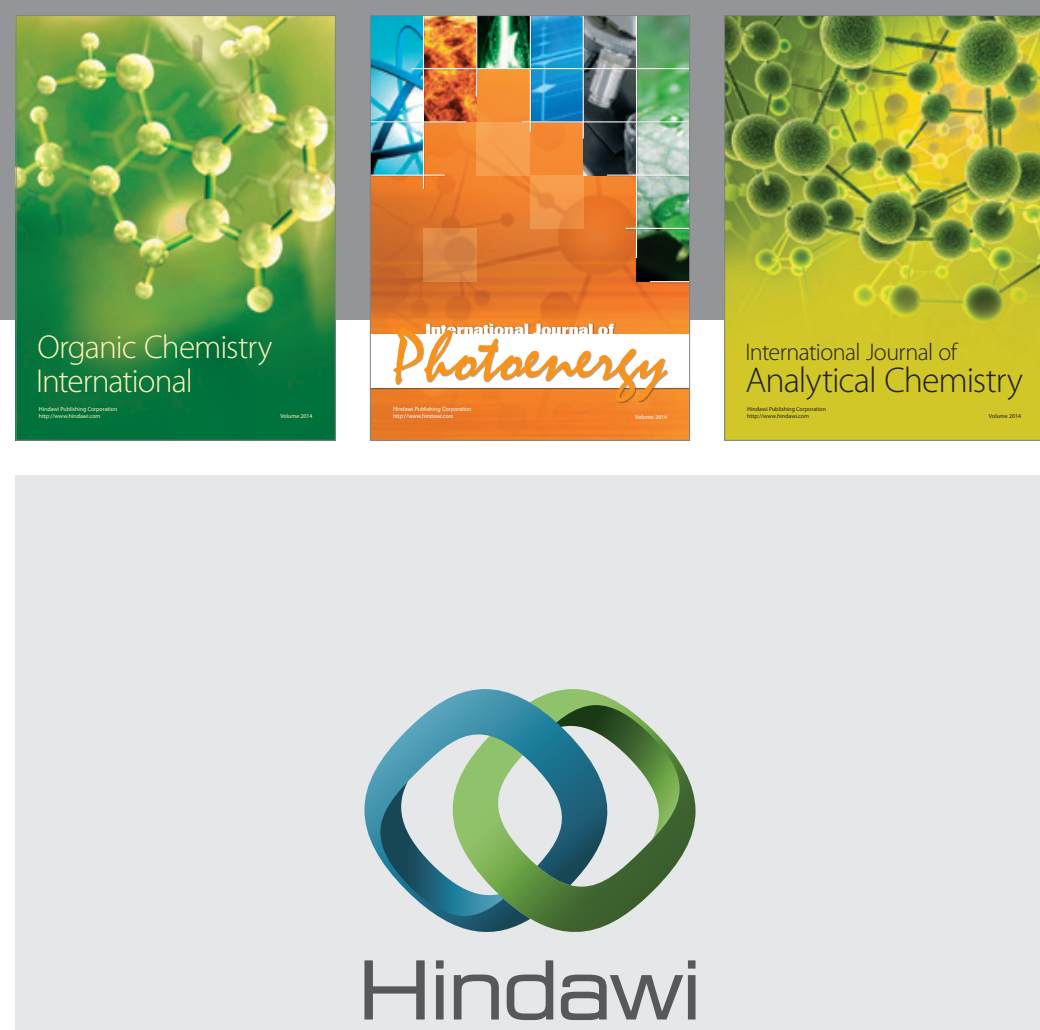

Submit your manuscripts at

http://www.hindawi.com
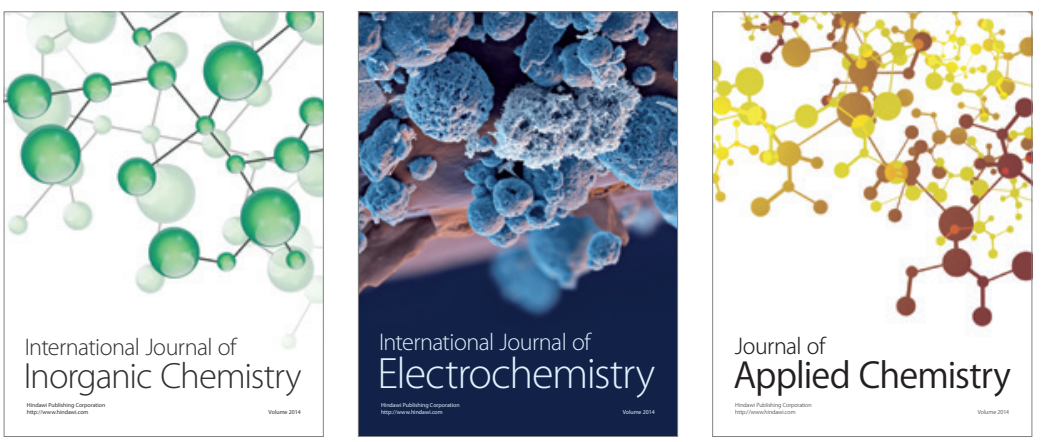

Journal of

Applied Chemistry
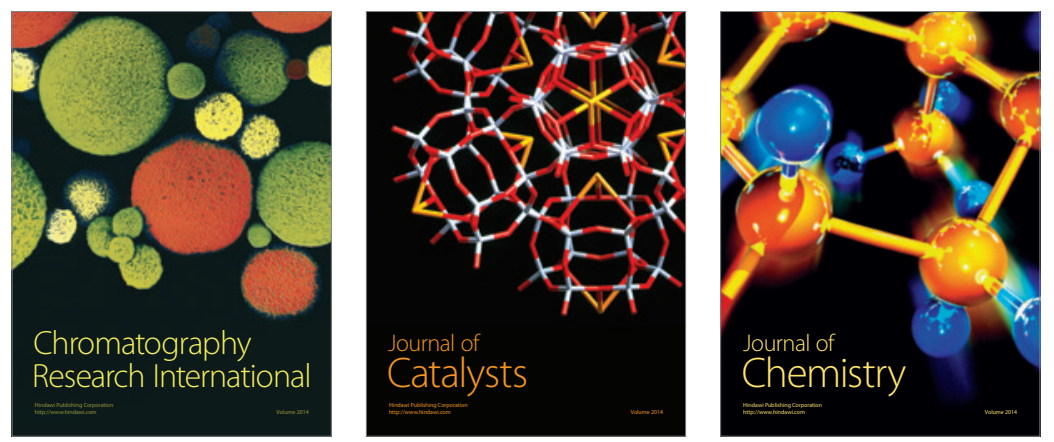
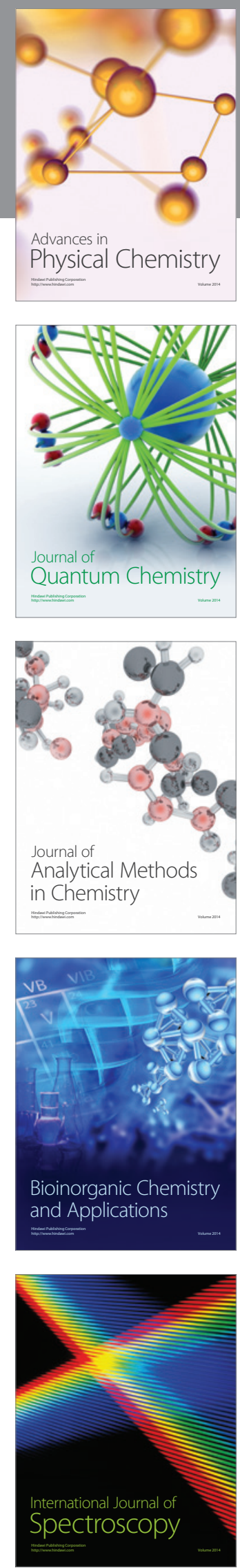